Project 1012275

\title{
Formation and Reactivity of Biogenic Iron Microminerals
}

\author{
Beveridge, Terrance J. \\ University of Guelph \\ Ferris, F. Grant \\ Univeristy of Toronto
}

\begin{abstract}
RESULTS TO DATE: Formation and Reactivity of Biogenic Iron Microminerals Interim Report
Terry Beveridge, Susan Glasauer and Anton Korenevsky, Department of Microbiology, University of Guelph
\end{abstract}

August 8, 2000

\section{Objective}

The overall purpose of the project is to explore and quantify the processes that control the formation and reactivity of biogenic iron microminerals and their impact on the solubility of metal contaminants. The research addresses how surface components of bacterial cells, extracellular organic material, and the aqueous geochemistry of the DIRB microenvironment impacts the mineralogy, chemical state and micromorphology of reduced iron phases.

1. General interfacial effects. During the past year we have continued to focus on processes at the interfaces between bacteria and fine-grained iron minerals, and bacteria and the aqueous environment. Scanning and transmission electron microscopy (EM) studies have shown that the minerals rapidly form a close association with the surface of some cells under aerobic and anaerobic conditions. Cells could not be separated from bound minerals by a step-gradient density method. EM observations on cells cultured with HFO under anaerobic conditions indicate that hydrophobicity of the outer membrane may influence the interaction.

We have made extensive studies to establish conditions for growth and for Fe reduction experiments that will more closely approximate natural conditions. This includes synthesis of fine-grained Fe minerals, which are typical of soil minerals, and acclimation of cell cultures (Shewanella putrefaciens CN32) to growth in a chemically-defined minimal medium.

2. Effect of lipopolysaccharides (LPSs) in biomineral production. LPS comprises about $40 \%$ of the mass of the outer membrane, extends from the cell surface to distances up to $\sim 40 \mathrm{~nm}$, and is a determining factor in the interaction of Gram-negative bacteria with environmental surfaces. It is an important biogeochemical structure since it has a particularly strong surface charge due to its abundant phosphoryl and carboxyl groups, and it actually participates in the development of fine-grained mineral phases. Information on the LPS composition of Shewanella is minimal; however, we would expect variations in LPS structure between Shewanella species and, therefore, in formation of different Fe minerals by distinct LPS types. Even differences in LPS composition in response to growth conditions (temperature, media composition, Eh and $\mathrm{pH}$ ) may influence the composition of biominerals.

We undertook experiments to establish the LPS composition of fifteen strains of iron-reducing Shewanella species, and determined the influence of temperature on LPS expression. This allowed us to choose strains with the greatest difference in LPS composition for biomineralization experiments. S. putrefaciens produces numerous membrane vesicles (MVs) during growth which possess many of the characteristics 
of the cell surface, including LPS. We have also characterized MVs of S. putrefaciens CN32 and S. oneidensis MR-1 to understand their interaction and function with iron oxide surfaces.

Approach for \#1. The fine-grained crystals (<30 nm) of hydrous ferric oxide (HFO), goethite (FeOOH) or hematite (Fe2O3) used in our studies were prepared by hydrolysis of $\mathrm{Fe}(\mathrm{III})$ salt solutions using sterile preparation methods. After crystallization was complete, the precipitates were washed four times followed by dialysis (goethite and hematite only) in sterile deionized water. The mineralogy was confirmed by x-ray and electron diffraction methods, whereas observations by TEM established the crystal size.

S. putrefaciens CN32 cultures used in initial anaerobic experiments were grown under aerobic conditions in tryptic soy broth (TSB), then transferred to a chemically-defined mineral medium (CDM) under anaerobic conditions. Because true anaerobiosis may not be achieved with this method and cells will not grow when directly transferred to CDM from TSB, cells were alternatively grown aerobically in CDM and then transferred to sealed tubes containing anaerobic CDM.

For studies on mineral adsorption by cells, Shewanella cultures were grown in TSB and then transferred to aerobic CDM. Fine-grained crystals of HFO, goethite or hematite were added and the suspensions were gently swirled for 30 minutes. Density gradient separations using step-glycerin gradients and centrifugation were done to isolate cells with sorbed minerals from mineral-free cells. By this method, the latter cells were less dense than $90 \%$ glycerin and, therefore, remained at the upper surface after centrifuging, while the high density of Fe-minerals caused Fe-sorbed cells to pellet at the bottom. We observed the associations by phase-contrast light microscopy, and by TEM using whole mounts (no stain) and thin sections from conventional embeddings and freeze-substitution preparations.

Adsorption of Shewanella added to a goethite-coated sand was followed by measuring protein in solution after incubating mixtures of cells plus prepared sand for 30 minutes. Sodium azide was added to some cell suspensions to inhibit growth and $\mathrm{H}+$ fluxes (which could affect sorption) before adding the cells to the goethite-sand. Samples were observed by scanning electron microscopy (SEM) after dehydration and gold-sputtering.

For an initial study of Fe reduction, cultures were grown aerobically on TSB, and then transferred to CDM in an anaerobic environment. Cysteine was added to some culture tubes before inoculation to determine the influence of redox potential on Fe reduction. TEM observations were made on thin sections prepared by conventional embedding.

Approach \#2. All bacterial strains were cultured aerobically at temperatures from $50 \mathrm{C}$ to $370 \mathrm{C}$ on either TSB or on TSB supplemented with $2 \% \mathrm{wt} / \mathrm{vol}$ of $\mathrm{NaCl}$ (i.e., for S. pealeana and S. woodyi). For SDSPAGE/silver staining, LPS was isolated by the proteinase $\mathrm{K}$ method.. For NMR, mass spectrometry and chemical analysis, LPS of S. putrefaciens CN32, S. oneidensis MR-1, and S. algae BrY were isolated by $\mathrm{MgCl}$-ethanol precipitation or by hot phenol method. For TEM, the bacterial cells were freeze-substituted and embedded. MVs were isolated from bacterial spent growth media and characterized by TEM, SDSPAGE, and differential redox spectrophotometry for cytochromes. MVs were also assayed for nitrate, fumarate and ferric iron reductase activities.

Progress for \#1. For the experiments on aerobic mineral sorption followed by glycerin separation, most of the cells were pulled to the bottom of the tube during centrifugation after exposure to the minerals; however, a fraction remained at the surface. The upper fraction contained cells with minerals that appeared tightly associated with the outer membrane. Samples preserved by freeze-substitution were particularly revealing; they showed minerals clustered at the outer periphery of intact cells, and, in many cases, penetrating the outer membrane. It often appeared that cells with sorbed minerals were surrounded by clean cells, which indicates that the surface physicochemistry differed between adjacent cells. 
It was difficult to quantitatively assess the ability of cells to sweep minerals from solution using conventional sorption isotherms; the relatively similar sizes of the two phases (minerals vs. cells), and the reactivity of the cells make their separation a challenge. To circumvent this, we used silica sand as a carrier for Fe oxide minerals, which form a strong bond to the silica surface under certain conditions. Solution concentrations of protein, indicative of free cells, were lower in the goethite sand treatment than in the clean sand control, indicating that more cells were associated with the goethite. We are attempting to confirm this using SEM/energy dispersive x-ray spectroscopy for Fe minerals beneath the cells. Cells exposed to sodium azide, which inhibits respiration, appeared to have less affinity for the goethite-sand. SEM studies showed that the sand grains were largely coated with goethite crystals, with bare patches on some smooth surfaces. Isolated cells appeared to be attached to the goethite, and goethite crystals that had dislodged from the sand were adhered to the bacteria.

Under anaerobic culture conditions (H2/Ar atmosphere), dissimilatory metabolism of HFO-Fe by Shewanella resulted in more than $85 \%$ Felll reduction. No well-defined crystalline mineral phases were detected by electron diffraction once Fe reduction had stopped. Cultures containing cysteine had lower redox potentials and showed less biotic Fe reduction.

We have found that cells appear to grow differently on TSB versus CDM with lactate as the electron donor. Shewanella cultures inoculated from TSB into CDM must be gradually introduced to CDM for growth to occur. This result is significant because most studies on dissimilatory Fe reduction by Shewanella use aerobic cultures of TSB-grown cells, which are inoculated into CDM under anaerobic conditions; by this procedure cells may maintain themselves on the stored metabolites accumulated under the TSB regimen and may not achieve true anaerobic metabolism. Our studies show that cells conditioned on CDM grow in aggregates. Examination of whole mounts by TEM revealed that the aggregated cells abutted each other at their outer membranes and no extracellular polysaccharide was seen. This mode of growth is more similar to what is found under natural conditions, in contrast to the well dispersed TSB-grown cultures of Shewanella. In addition, cells grew much more slowly in CDM and populations at the peak of growth were several orders of magnitude lower than were observed with TSB. They were, however, still capable of mineral Fe reduction.

Progress for \#2. Silver-stained SDS-PAGE gels demonstrated that all examined strains express some rough LPS. S. putrefaciens, S. oneidensis, S. baltica, Shewanella sp. MR-4, and Shewanella sp. CL $256 / 73$ produced only rough LPS, whereas S. algae, S. amazonensis, S. frigidimarina, S. pealeana, and S. woodyi produced some high-molecular weight smooth LPS.

Growth temperature markedly influenced the LPS core oligosaccharide/O-sidechain composition in some strains. Temperatures below $250 \mathrm{C}$ resulted in expression of semi-rough LPS. Maximum size heterogeneity of LPS was observed at $15-200 \mathrm{C}$. This was most apparent in the case of S. frigidimarina where a characteristic ladder-like banding pattern appeared. A further decrease in growth temperature caused a decrease in LPS size heterogeneity. However, O-sidechain expression by the majority of strains was usually not affected by temperature.

Because freeze-substitution preserves the extended conformation of LPS O-sidechains, it was of interest to see if we could confirm the results of our SDS-PAGE by TEM. Thin sections of semi-rough $S$. oneidensis MR-1 revealed a fibrous fringe surrounding cells extending $\sim 10-12 \mathrm{~nm}$ from the cell surface, while the cell surface of rough S. putrefaciens and S. baltica were devoid of any fibrous material. Interestingly, the presence of high-molecular weight LPS in S. algae and S. amazonensis did not result in a visible fibrous layer in their thin-sectioned cells. This could be due to the chemistry of the LPS Osidechains since they may be composed of neutral sugars.

It was shown by NMR, mass spectrometry and chemical analysis that S. putrefaciens CN32 LPS was unusually homogenous and contained no structural variants in detectable amounts. Another unusual feature was the substitution of ketodeoxyoctonate(KDO) with a DD-Hep residue. D-glycero-D-manno-Hep is a biosynthetic precursor of LD-Hep, thus $\mathrm{S}$. putrefaciens CN32 may have no mechanism for conversion of DD-Hep to LD-Hep. 
Isolated MVs from S. putrefaciens CN32 and S. oneidensis MR-1 had an average diameter of 30-100 nm. The MVs of both strains possess rough LPS as determined by silver-staining SDS-PAGE and exhibited lipase and protease activities. No nucleic acids were detected. Differential spectra (red/ox) indicated the presence of c-type cytochromes in MVs, and SDS-PAGE heme-staining revealed cytochromes with $\mathrm{Mr}=$ $78.2,72.4$, and 66.1 kDa in CN32 MVs, and 75.8 and $66.5 \mathrm{kDa}$ in MR-1. Dithionate-reduced cytochromes in MVs were oxidized under anaerobic conditions by ferric iron, nitrate and fumarate, according to the benzyl vilogen assay and spectral changes. KDO/protein ratios were quite low in Shewanella MVs (0.0034 and 0.0033 in MR-1 and CN32, respectively) compared to 0.02 for Pseudomonas aeruginosa PAO1, apparently due to Shewanella's KDO substitution in LPS and the high protein content in MVs.

Future work for \#1. We will continue to study anaerobic Fe reduction in CDM using our collection of finegrained Fe minerals. The focus will be on reduction products and the spatial relationship of cells to the dissolving mineral and to any newly formed mineral phases; both Shewanella and Geobacter cultures will be used for these studies. In particular we will study the relationship between cell age or metabolic state and mineral sorption.

Fe-oxide coated sand will be used for further studies on cell-mineral interactions. Selected treatments to disable membrane function will help in understanding the influence of metabolism on sorption.

Examination of the cell-mineral interface will determine the closeness and nature of the attachment, i.e., via extracellular polysaccharides or other mechanism. The use of membrane vesicles, which contain only outer membrane, will allow us to focus on the influence of surface properties on mineral sorption.

The anaerobic growth of Shewanella as biofilms will be investigated in columns of Fe oxide coated sand under aerobic and anaerobic systems. Such flow-through systems are a better analog to natural conditions than batch culture methods, and such a system can be easily manipulated to study common environmental fluxes, such as starvation/nutrition cycles and the introduction of toxins such as heavy metals.

Future work for \#2. We will continue to characterize Shewanella's MVs, but the focus will be on the redox properties of its cytochromes, and cytochrome distribution in MVs with different sizes. Also, we will study the influence of culture age on the cytochrome content in MVs.

Species with three different types of LPS: rough, semi-rough and smooth, i.e, S. putrefaciens CN32, S. oneidensis MR-1, and S. algae BrY will be tested for metal sorption (particulary Ni, U, Fell ,and CrIII). These strains will also be tested for possible selectivity in sorption of different types of iron minerals by the cells; in addition, the influence of LPS on the composition of minerals developing on the cell surfaces will be investigated. Studies on hydrophobicity/hydrophilicity and electrophoretic mobility of the cells will give additional information on surface properties that may influence metal and iron mineral sorption.

DELIVERABLES: Beveridge, T. J. Keynote speaker, Strong, tough and elastic: bacterial cell walls as nucleation sites for developing fine-grained minerals. Geol. Society America Conference, Denver, CO, Oct. 25, 1999.

Beveridge, T. J. Seminar, Department of Geosciences, Princeton, U., Nov. 22, 1999.

Beveridge, T. J. Seminar, Department of Microbiology, U. of New Hampshire, Durham, NH., Feb. 28, 2000.

Beveridge, T. J. Keynote speaker, Geomicrobiology: making and breaking minerals, Amer. Soc. Microbiol. Annual Meeting, Los Angeles, CA, May 23, 2000.

Beveridge, T. J. Invited speaker, Microorganisms in geology: finding common ground, GeoCanada 2000 (combined meeting of Geological Societies), Calgary, AB, Canada, May 30, 2000. 
Glasauer, S. Invited speaker, Surface-mediated metal precipitation by bacteria, Speciation of Elements in Biological, Environmental and Toxicological Sciences, 4th Intl. Symposium, Whister, BC, Canada, June 27, 2000.

Korenevsky, A. A. and Beveridge, T. J. Influence of temperature on lipopolysaccharide expression in Shewanella (poster), Amer. Soc. Microbiol. Annual Meeting, Los Angeles, CA, May 24, 2000. 